\title{
Phonon dispersion relations and densities of states in $\mathrm{La}_{2} \mathrm{CuO}_{4}$ and $\mathrm{La}_{2} \mathrm{NiO}_{4}$
}

\author{
NARAYANI CHOUDHURY, K R RAO and S L CHAPLOT \\ Solid State Physics Division, Bhabha Atomic Research Centre, Bombay 400085, India.
}

\begin{abstract}
Rigid-ion model calculations of phonon dispersion relations, densities of states and partial densities of states of the high $T_{c}$ superconductor $\mathrm{La}_{2} \mathrm{CuO}_{4}$ and its isostructural compound $\mathrm{La}_{2} \mathrm{NiO}_{4}$ have been carried out both in the tetragonal and orthorhombic phases of $\mathrm{La}_{2} \mathrm{CuO}_{4}$ and the tetragonal phase of $\mathrm{La}_{2} \mathrm{NiO}_{4}$. The calculations are in fair agreement with reported experiments. The computed phonon dispersion in the tetragonal phase of $\mathrm{La}_{2} \mathrm{CuO}_{4}$ reproduces the soft mode behaviour for the lowest $\Sigma_{4}$ TO branch which is found to harden in the orthorhombic phase, consistent with experimental data.
\end{abstract}

Keywords. $\mathrm{La}_{2} \mathrm{CuO}_{4} ; \mathrm{La}_{2} \mathrm{NiO}_{4}$; phonon dispersion relations; densities of states.

\section{Introduction}

We have carried out the study of lattice dynamics of $\mathrm{La}_{2} \mathrm{CuO}_{4}$ and its isostructural compound $\mathrm{La}_{2} \mathrm{NiO}_{4}$. Our objective has been to calculate the phonon dispersion relations, densities of states and partial densities of states and compare these results with the available experimental data. Calculations have been undertaken for both tetragonal and orthorhombic phases of $\mathrm{La}_{2} \mathrm{CuO}_{4}$ and the tetragonal phase of $\mathrm{La}_{2} \mathrm{NiO}_{4}$. We have aimed at understanding the differences in the phonon spectra in going from the tetragonal to the orthorhombic phase and the similarities in the phonon dispersion in the tetragonal structure of $\mathrm{La}_{2} \mathrm{CuO}_{4}$ and $\mathrm{La}_{2} \mathrm{NiO}_{4}$. The computed phonon dispersion relations and densities of states in these compounds are found to be in fair agreement with experimental data.

\section{Lattice dynamical model}

The lattice dynamical calculations are carried out within the scope of an unscreened rigid-ion model in the quasiharmonic approximation. A detailed justification for using an unscreened model for the lattice dynamical studies on $\mathrm{YBa}_{2} \mathrm{Cu}_{3} \mathrm{O}_{7}$ has been reported by Chaplot (1988). The interaction potential consists of Coulombic and short-ranged terms and the potential energy between two non-bonded atoms $k$ and $k^{\prime}$ separated by a distance $r$ is given by

$$
V(r)=\left(\frac{e^{2}}{4 \pi \varepsilon_{0}}\right) \frac{Z(k) Z\left(k^{\prime}\right)}{r}+a \exp \left\{\frac{-b r}{R(k)+R\left(k^{\prime}\right)}\right\},
$$

where $R(k)$ and $Z(k)$ are the effective radius and charge parameters, respectively, of the atom $k$. The parameters are evaluated using equilibrium conditions of vanishing stress and forces on all the atoms for the given structure and are discussed in detail in Choudhury et al (1990). The same parameters have been used for the calculations in both phases of $\mathrm{La}_{2} \mathrm{CuO}_{4}$. 
(a)

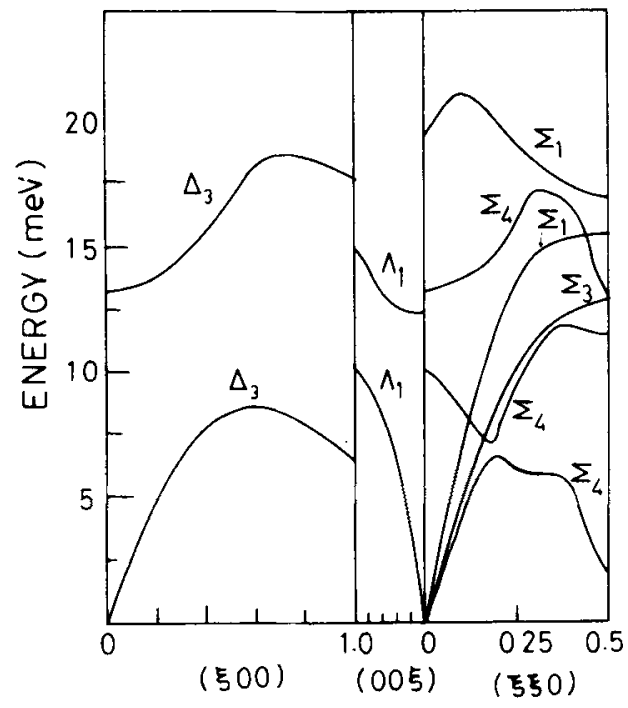

(b)

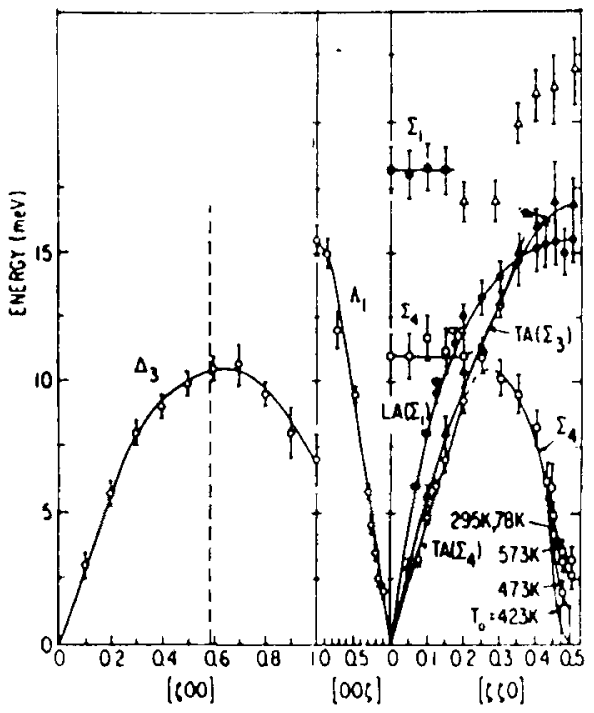

Figure 1. Comparison of (a) the computed phonon dispersion relation along the three high symmetry directions in the tetragonal phase of $\mathrm{La}_{2} \mathrm{CuO}_{4}$ with (b) available experimental data (Birgeneau et al 1987).

\section{Results and discussion}

The computed long wavelength phonon frequencies in $\mathrm{La}_{2} \mathrm{CuO}_{4}$ and $\mathrm{La}_{2} \mathrm{NiO}_{4}$ are in good qualitative agreement with Raman and infrared data; however the computed LO-TO splittings are not anisotropic as observed in infrared experiments, suggesting the need for an anisotropic screening term in the interaction potential. Calculations using a 'screened rigid-ion model' are currently in progress.

The computed phonon dispersion relation in $\mathrm{La}_{2} \mathrm{CuO}_{4}$ (figure 1) and $\mathrm{La}_{2} \mathrm{NiO}_{4}$ are found to be in fair agreement with experimental data (Birgeneau et al 1987; Pintschovius et al 1988) and the computed $\Sigma_{4}$ mode in the tetragonal phase of $\mathrm{La}_{2} \mathrm{CuO}_{4}$ exhibits soft mode behaviour, which is hardened in the orthorhombic phase. The eigenvector of the soft mode is consistent with the observed orthorhombic distortion.

Almost all the branches are slightly shifted to higher frequencies in the orthorhombic phase as compared to those in the tetragonal phase of $\mathrm{La}_{2} \mathrm{CuO}_{4}$ although the nature of phonon dispersion remains almost identical in the two phases. The computed phonon dispersion relation in $\mathrm{La}_{2} \mathrm{NiO}_{4}$ is qualitatively similar to that observed in $\mathrm{La}_{2} \mathrm{CuO}_{4}$.

The computed 'neutron-weighted' densities of states in these compounds (figure 2) are similar to those observed experimentally (Renker et al 1987), but the peaks in the computed densities of states are systematically shifted to lower frequencies. The computed partial density of states (figure 3) have helped in understanding the anisotropy in the dynamical contributions to frequency spectrum arising from various species of atoms namely, the $\mathrm{La}, \mathrm{Cu}$ and the inequivalent oxygen atoms $\mathrm{O}_{1}$ and $\mathrm{O}_{2}$. 


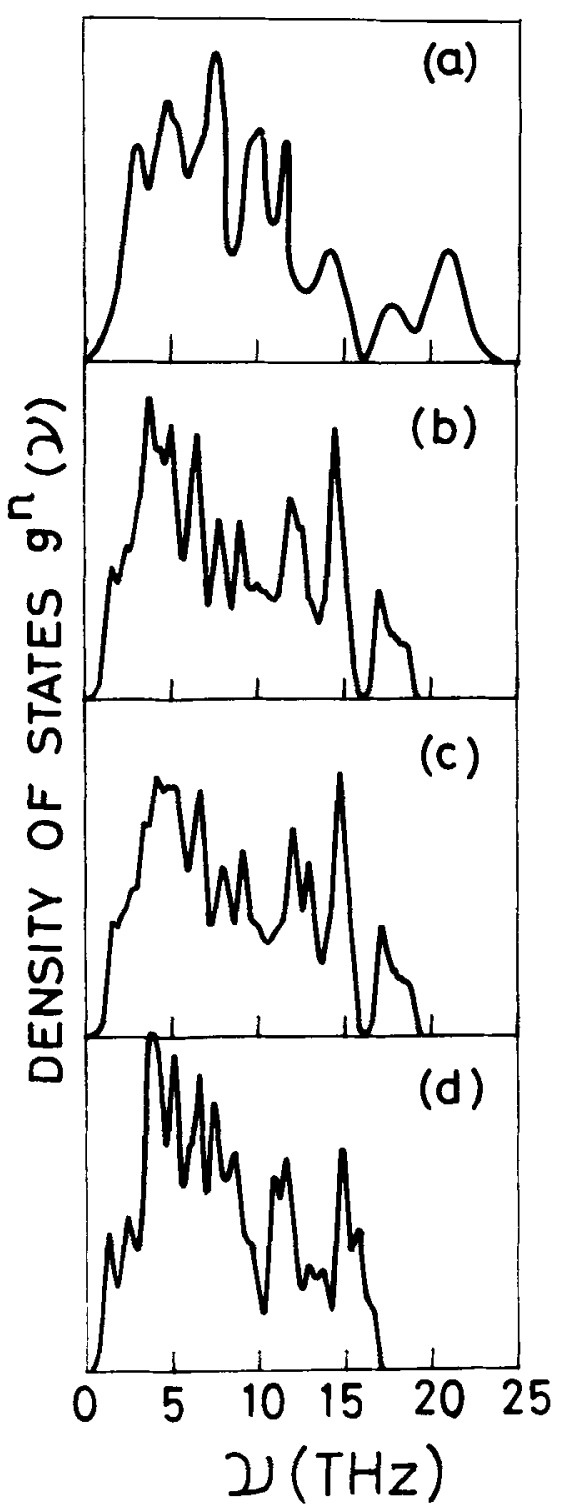

Figure 2. Comparison of the experimental density of states of Renker et al (1987) in (a) with our computed neutron-weighted densities of states in the orthorhombic and tetragonal phases of $\mathrm{La}_{2} \mathrm{CuO}_{4}$ and the tetragonal phase of $\mathrm{La}_{2} \mathrm{NiO}_{4}$ in (b), (c) and (d) respectively.

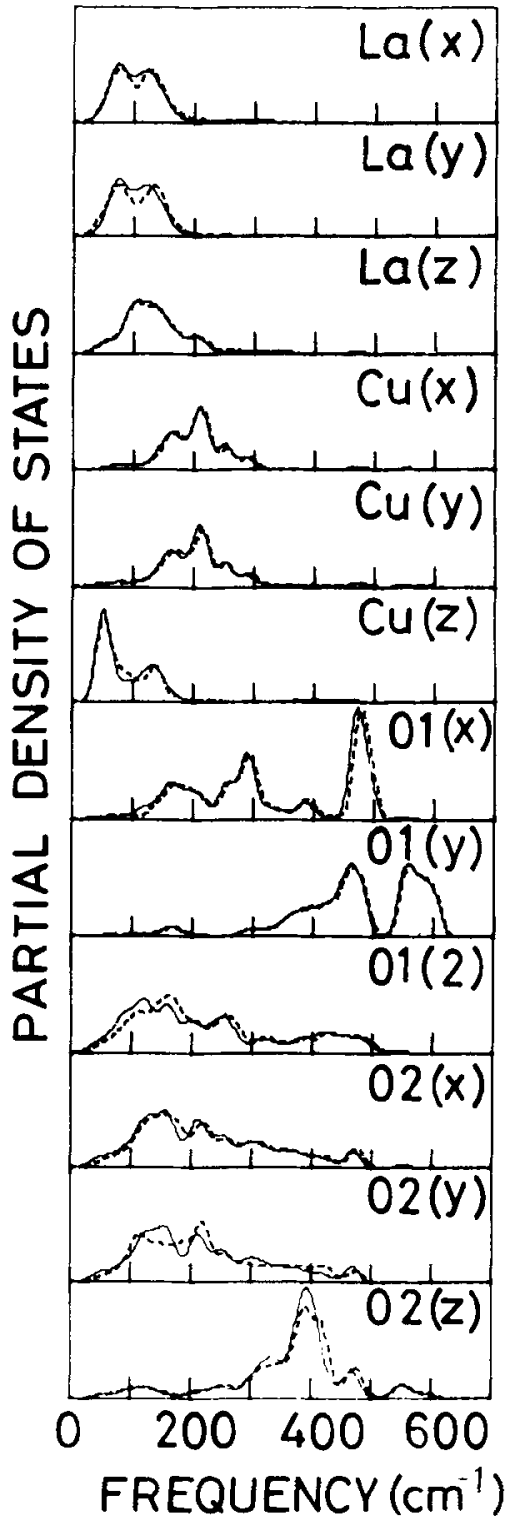

Figure 3. Computed partial densities of states in the tetragonal (full line) and the orthorhombic phases (dashed line) of $\mathrm{La}_{2} \mathrm{CuO}_{4}$. 
Detailed comparison of our results with those reported by others, especially by Pintschovius et al (1989), since submission of the abstract to this conference, will be published elsewhere.

\section{References}

Birgeneau R J et al 1987 Phys. Rev. Lett. 591329

Chaplot S L 1988 Phys. Rev. B37 7435

Choudhury N, Rao K R and Chaplot S L 1990 Physica C171 567

Pintschovius L, Bassat J M, Odier P, Grevais F, Hennion B and Reichardt W 1988 Europhys. Lett. 5247

Pintschovius L, Bassat J M, Odier P, Grevais F, Chevrier G, Reichardt W and Gompf F 1989 Phys. Rev. B40 2229

Renker B, Gompf F, Gering E, Nucker N, Ewert D, Reichardt W and Rietschel H 1987 Z. Phys. B67 15 